\title{
3D MODELING OF SATBAYEV UNIVERSITY BASED ON LASER SCANNING AND UAV DATA
}

\author{
B. Adebiyet ${ }^{1}$, F.A. Iliuf ${ }^{2}$, E. Orynbassarova ${ }^{3}$, A. Chernov ${ }^{4}$, K. Idrissov $5 *$ \\ ${ }^{1}$ KazNITU, Mining, and Metallurgical Institute. O. A. Baikonurova, Almaty, Kazakhstan - astbaha6@ gmail.com \\ ${ }^{2}$ KazNITU, Mining, and Metallurgical Institute. O. A. Baikonurova, Almaty, Kazakhstan - fatimkaa99@ gmail.com \\ ${ }^{3}$ KazNITU, Mining, and Metallurgical Institute. O. A. Baikonurova, Almaty, Kazakhstan - \\ e.orynbassarova@satbayev.university \\ ${ }^{4}$ KazNITU, Mining, and Metallurgical Institute. O. A. Baikonurova, Almaty, Kazakhstan - a.chernov@ satbayev.university \\ ${ }^{5}$ Leica Geosystems Kazakhstan, Almaty, Kazakhstan - Kirill.Idrissov@ leica-geosystems.com
}

KEYWORDS: laser scanning, point cloud, post-processing algorithm, UAV, 3D model, GNSS, Satbayev University

\begin{abstract}
:
The article describes one of the fastest growing and important areas of information technology - the 3D modeling of buildings and structures. In particular, the results of the work performed when creating a three-dimensional model of Satbayev University, which is the first technical university in the Republic of Kazakhstan, are considered. The main goal of the work is to create an interactive threedimensional model of the university building based on laser scanning data and aerial photography from an unmanned aerial vehicle (UAV). A detailed 3D model was built by combining images taken from the DJI Phantom 4 Pro UAV and a dense point cloud obtained from the Leica RTC360 laser scanner. Based on the work performed, the advantages and disadvantages of the integrated use of these techniques were evaluated. The results obtained are planned to be integrated with AR / VR technologies, BIM, GIS, and web mapping.
\end{abstract}

\section{INTRODUCTION}

Satbayev University is the first technical university in Kazakhstan, it was built in 1934 in Almaty. The object is of historical value and in addition, is the center of scientific and pedagogical development of the Republic. The tallest building in the university has a height of nine floors. The authors set the task of complete detailing of the outer shell of the building, which should be achieved by creating a high-precision threedimensional model.

In recent years, the construction industry has made great strides through the combination of automation and information technology. Many methods have been actively developed due to the growing demand for 3D modeling of spatial information. Particular attention was paid to the technology of joint use of laser scanners and unmanned aerial vehicles to create a point cloud in 3D modeling at construction sites. In turn, the point cloud serves as the basic basis for exporting to BIM / GIS and the formation of three-dimensional models of objects.

UAVs are one of the optimal means for creating a point cloud. This is the most cost-effective and at the same time accurate source of obtaining spatial data used to change the shape of buildings during surveys. A 3D building model is reconstructed with symmetry in mind - by creating interconnected surfaces and using geometric relationships between structural elements.

The following is a technique for post-processing the results of laser scanning and UAV. The creation of a detailed 3D model based on a processed point cloud is considered in detail.

\section{INVESTIGATION OF AN OBJECT WITH THE HELP OF A UAV}

\subsection{UAV shooting technique}

Using aerial photography technology (using UAV), you can perform an accurate photogrammetric survey in a short time, as well as create an accurate 3D model.

Speaking about the use of UAVs in cartography and creating $3 \mathrm{D}$ models of buildings, the following advantages should be noted (Service Geo, 2014):

$\checkmark \quad$ high profitability in comparison with traditional survey methods;

$\checkmark \quad$ speed of obtaining photographs, DSM and DEM;

$\checkmark \quad$ obtaining high-resolution photographs due to the possibility of flying from low altitudes.

The complex geometry of buildings and the presence of hardto-reach places (trees, wires, etc.) in the areas being filmed creates "blind zones" for shooting UAVs, which is ultimately reflected in the quality of the created model.

For aerial photography, a DJI Phantom 4 Pro V2 UAV was used (fig.1).

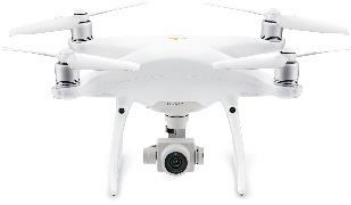

Figure 1. DJI Phantom 4 pro V2.

Cross-corridor shooting mode was chosen as the shooting mode, which makes it possible to form a larger number of mutually overlapping images, which is important for increasing the detail of the created model. Taking into account the heights of nearby objects, the following parameters of the flight task were set - the flight altitude was set to $80 \mathrm{~m}$ (taking into account the height of the object of study, neighboring structures, and compliance with flight safety); the camera tilt angle is set to 750 (to capture the lower floors of the object). 
The total shooting time was 25 minutes. The received materials (fig.2.) were further processed in the Pix4D mapper software.

\subsection{Using GNSS Surveys}

GNSS survey is a satellite positioning technique that uses GPS/Glonass/BeiDou/Galileo satellite systems to locate unknown points (Kuzmenko S.V., Shamganova L.S., Akhmedov D.Sh., Baltieva A.A.,2018).

In our case, the GNSS survey was used to reference aerial photography materials. The control points were captured using a Leica GS-16 GNSS receiver in real-time, which allows you to receive corrections to measurements and set the location with centimeter accuracy. The coordinate system was used local (MSK Almaty). The accuracy of determining the reference points in the plan was $1 \mathrm{~cm}$, in height $-2 \mathrm{~cm}$. In total, twenty-two reference points were captured.

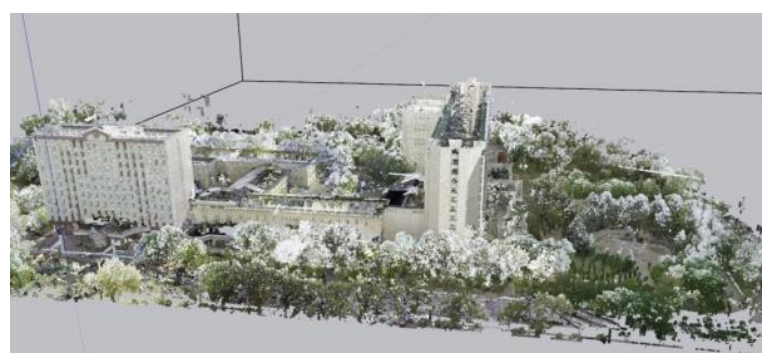

Figure 2. Satbayev University point cloud.

\subsection{Algorithm for processing the received materials in the Pix4D mapper software}

Processing of images obtained from the UAV was carried out in the Pix4D mapper software version 4.6.4. It is the most suitable software for photogrammetry (YUNEEC, 2019), which can generate ortho-mosaic and 3D point cloud data with high accuracy. The program automatically extracts a large number of tie points on each photo.

Consider the processing algorithm we use. At the beginning of the work, a new project is created. We load the image into the created project, where we set the following parameters: coordinate system, geopositioning and orientation of images, geolocation accuracy, camera model, and interior orientation elements (fig.3).

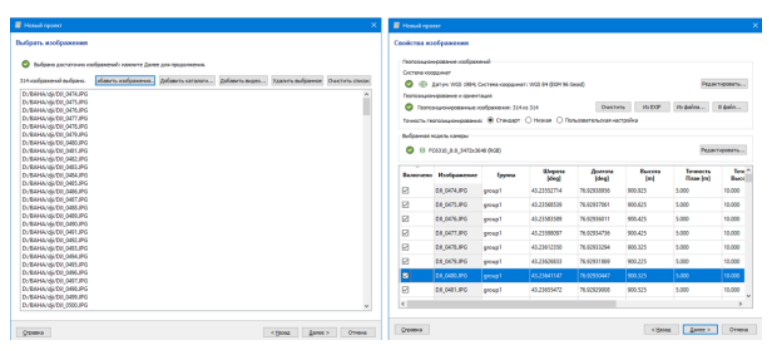

Figure 3. Loading images.

The next step was to select the output coordinate system for our project, in our case it is the local coordinate system of the city of Almaty. Then select the processing type in the processing parameters window. The program has three types to choose from 3D maps, 3D models, and multispectral. Since we needed to get a 3D model, we chose the "3D models" type accordingly. As a result, we got a 3D surface and a cloud of points. If necessary, it was also possible to obtain an orthophoto map and a digital surface model (DSM). Next, the program loads the images and the flight route. The next step is to open the GCP/tie point manager window to define and bind GCPs. Using the "import anchor points" tab, we add points in the .txt format. After that, each control point was corrected using images and a report was received on the work performed. The report shows Dewarping options related to the electronic shutter; accuracy of binding control points; "Rolling shutter" adjustment, which allows you to compensate for the speed of the drone's camera movement relative to the ground.

The next step is the launch of the processing stage, which consists of the following parts: initial processing, point cloud, surface, DTM, orthomosaic, and indexes. After completion of all stages, a report was uploaded on the processes performed and on the quality of the data received. To view the results, there are several tabs on the left side of the program panel; in the orthomosaic editor, you can view the constructed orthomosaic, point cloud, and 3D model (fig.4).

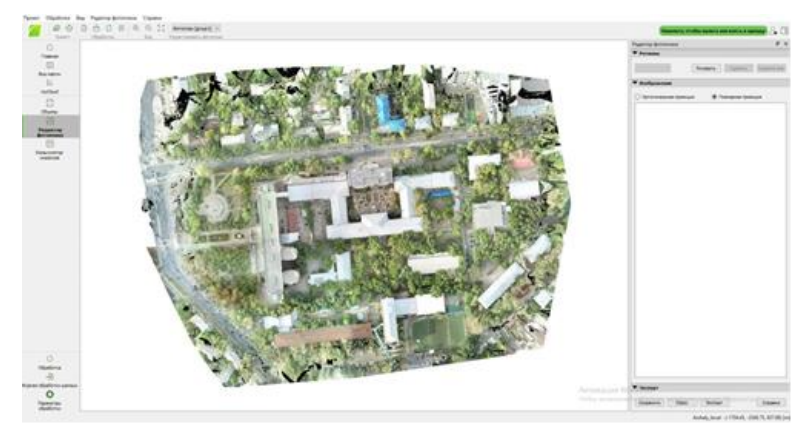

Figure 4. Orthophoto map.

In the ray Cloud section, you can see the results for the point cloud, control points, model, and triangulation surface (fig.5).

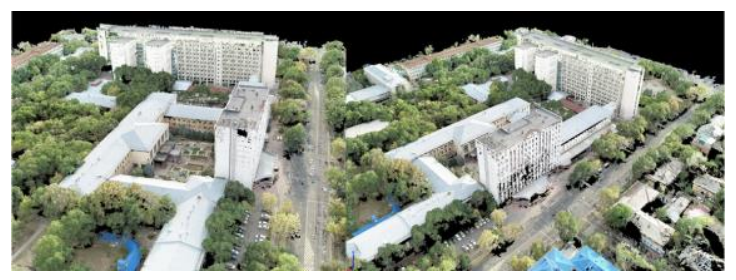

Figure 5. University model according to UAV data.

As a result, an ortho-image, a cloud of points, and a 3D model were automatically created based on a comparison of the characteristic points of a set of images. Point cloud data containing location information and elevation data has been output, and a 3D model has been created. The accuracy of the 3D model and DSM (Digital Surface Model) has also been verified with this software. Taking into account the shooting time of 25 minutes and the time spent on processing - 4 hours, the results obtained are quite satisfactory for the rapid creation of a model.

\section{INVESTIGATION OF AN OBJECT USING TERRESTRIAL LASER SCANNING}

\subsection{Development of a 3D model using laser scanning}


Laser scanning is one of the modern methods of detailed three-dimensional modeling. The choice of the Leica RTC 360 laser scanner model was justified by several advantages, new innovative features (A. A. Baltiyeva, A. S. Raskaliyaev, A. I. Samsonenko, L. S. Shamganova, H. Fan., 2020).

First, the tracking system VIS (visual-inertial system) tracking the location of the scanner in real-time. This system allows you to automatically register the scanner in the field in real-time. Registration is intended to identify the coordinates of the place where the laser scanner will be located. It is possible to select a new station for scanning and at the moment when the scanner moves, it already determines its coordinates through VIS calculations.

Second, the Leica RTC 360 (Copyright Leica Geosystems AG, 2017) can capture 2 million points per second and has a range of 130 meters.

Thirdly, the average scanning time at one station is 1 minute 50 seconds with photographing the area.

Fourth, the horizontal field of view is 3600 and the vertical is 2700 .

Fifth, IP54 with a fully enclosed mirror - which means it can work in rain, snow, or dust.

Sixth, the scanner has two ways to remove noise. It will scan twice and if there are points that don't exist in both point clouds - the software will automatically delete them.

Before the start of the survey, the following parameters were set: the density of points - 6 by $6 \mathrm{~mm}$ (for greater detail of the area), and for the monument named after K.I. Satpayev point density was increased by 2 times. The implementation of the survey, which consisted of 124 stations, took 6 hours.

\subsection{Algorithm for processing the received data in the Cyclone register software}

After the survey is completed, the data obtained are imported into the Cyclone register program, where the registration stage takes place (fig. 9). Leica Cyclone REGISTER 360 is the latest upgrade product of Cyclone REGISTER, the best point cloud data synthesis processing software. This new product offers an entirely new set of features built from the ground up with a simple, guided workflow. Intuitive quality control tools and reporting capabilities ensure you can determine the quality of your composite data, not guesswork, and have all the information you need at your fingertips. From site to final operation Cyclone REGISTER 360 is part of an integrated RTC360 solution. The Cyclone REGISTER 360 uses the RTC360 Visual Inertial System (VIS) to automatically place collected data into coordinate values and complete registration with minimal user effort. The collected project data will be published as a new Leica Geosystems Universal (LGS) project file for use with all HDS software products. One file offers endless possibilities.

Before starting work, a database was created in the software into which the completed survey is imported. The point cloud has its internal format, it optimizes fast rendering through compression. In our case, the volume was 8 gigabytes. The registration stage includes the point cloud optimization process. In case of gross inconsistencies between groups of the point cloud in the program, you can "manually" move it to the correct location and optimize the overlap and communication compatibility between stations (fig.6).

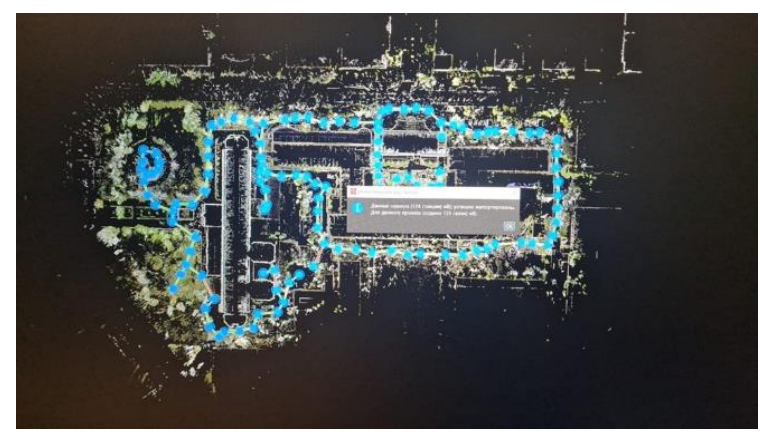

Figure 6. The process of registering a new project.

With the worst alignment of point cloud groups, they will be displayed in red, with an acceptable value in yellow, with the best alignment in green (fig.7). After that, the program will automatically recalculate the optimization parameters.

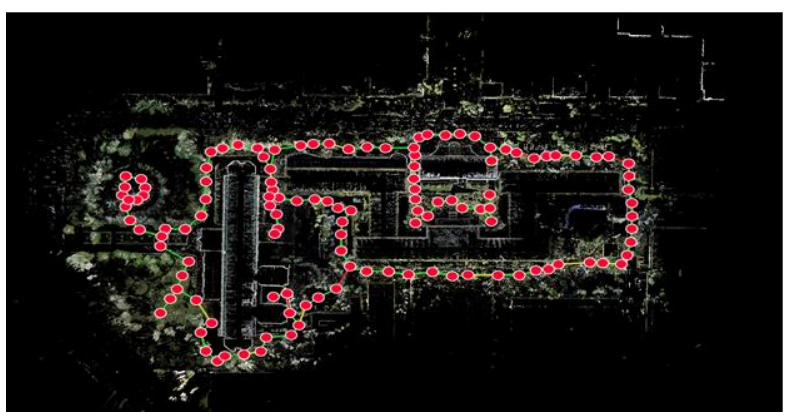

Figure 7. The process of optimizing point cloud groups.

Next in the program, you need to open each line called "link" between the stations and check the horizontal and vertical compatibility. If the point cloud groups are not displayed correctly, then using the "move" function you need to move it to the correct location (fig.8).

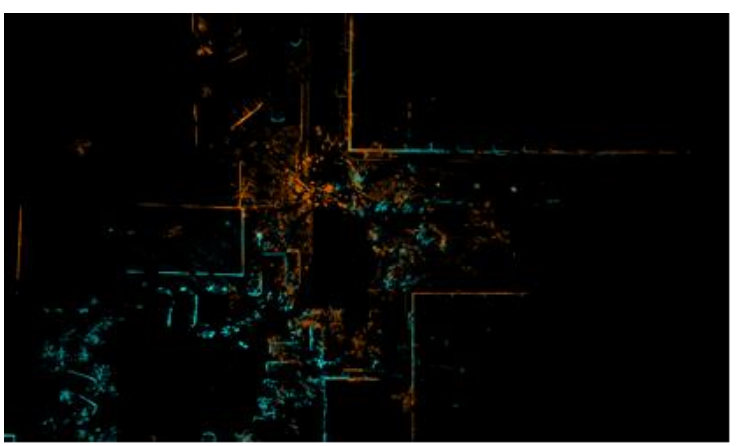

Figure 8. Incompatibility of point cloud groups.

Successful completion of the registration phase is confirmed by a progress report. It consists of the results of the total error, which is $-4 \mathrm{~mm}$, overlaps, compatibility between point cloud groups (fig.9). 


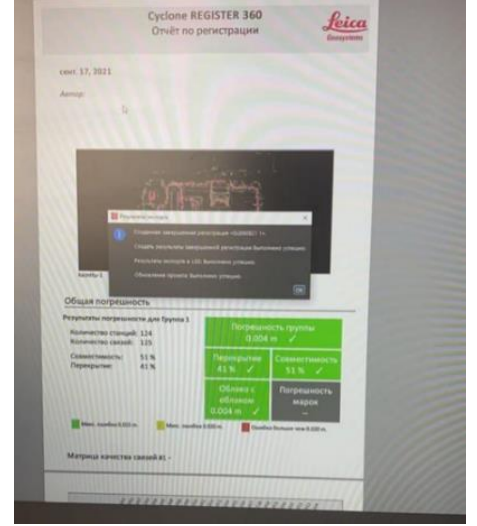

Figure 9. Completing the registration process.

Each station was cleaned from noise. In our case, the noise was: erroneous points located outside the area of the study area (people, cars, etc.). Removing such points lightened the amount of data and optimized fast visualization.

\subsection{Point cloud anchor}

Control points were loaded into the Cyclone program, which was captured using the Leica GS16 GNSS receiver in .txt format. Each point is manually coordinated into the point cloud. The program automatically optimized the binding and calculated the standard deviation. In our case, the standard deviation is $5 \mathrm{~cm}$, which is sufficient accuracy for further modeling. If necessary, you can reproject the data. The final step is to export the point cloud. The point cloud has been converted to .las from the internal format. lgs, because for further creation of 3D models, SketchUp software, which supports it, will be used. las files.

\subsection{Processing of received materials in SketchUp software}

Nowadays, there are many different programs. But just for solving problems in $3 \mathrm{D}$ modeling, based on a cloud of points, they can be counted on the fingers. When choosing a program, first of all, well-known, but not for everyone, available software immediately comes to mind, both in terms of price and interface. It is very difficult to master such programs as AutoCAD Revit, Blender, Avevo without special training. It is advisable to use such software in design institutes. SketchUp software was chosen to solve our problem.

SketchUp is characterized by easy-to-use functionality and intuitive operations. The main steps of the operation start with drawing lines and surfaces, building a solid from lines and surfaces, and then modeling by combining solids. SketchUp $3 \mathrm{D}$ also makes it easy to share designs.

Undet for SketchUp is a plug-in that is not included in the standard version, it extends the additional feature of cloud data points, providing the power and flexibility to use large amounts of cloud data. The Undet point cloud engine maintains the quality and accuracy of the model and can process billions of points without high loss. Creating 3D geometry from point snaps in SketchUp simplifies the workflow from scanning to modeling and greatly increases the productivity of $3 \mathrm{D}$ models. The module itself supports the import of various point cloud data from major scanner manufacturers such as Leica, Riegl, FARO, Topcon, as well as various point cloud data in las, ASCII format (fig.10). To work, we asked the official representatives of Undet, a free student license, which gives access to three months of unhindered use (Undet for SketchUp Development Team, 2020).

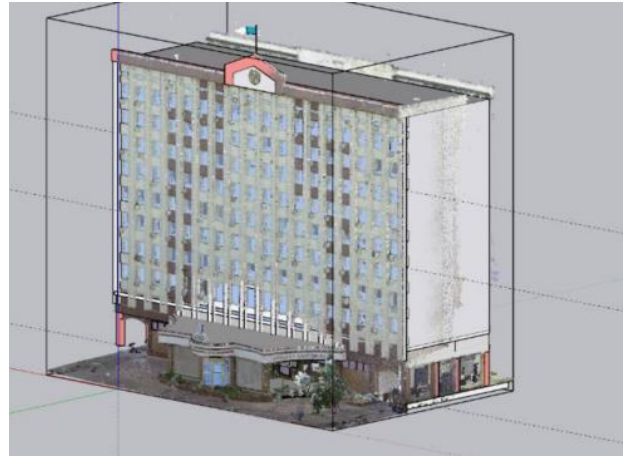

Figure 10. Point cloud in SketchUp software loaded using the Undet module.

The program with the connected Undet module has advanced functions for working with point cloud data. For the convenience of drawing, the program has a segment clipping function. You can select only the desired section of the point cloud that interests us, and the rest of the data will not be displayed temporarily, here you can also adjust the density of the display of points, this is very convenient when you work with a large amount of data. There are functions for moving and rotating point cloud data. There are several customizable views such as top, bottom, left, right. All these features allow you to display data optimally according to the quality and condition of the point cloud data.

The program has a shading function. The function itself has several kinds of shading, such as adjusting the contrast, RGBbased brightness, and display intensity, which are contained in the point cloud data. You can set the step color to be displayed according to a range of distances such as height and depth (fig.11). It is also possible to set the display of the natural shape of the point cloud data.

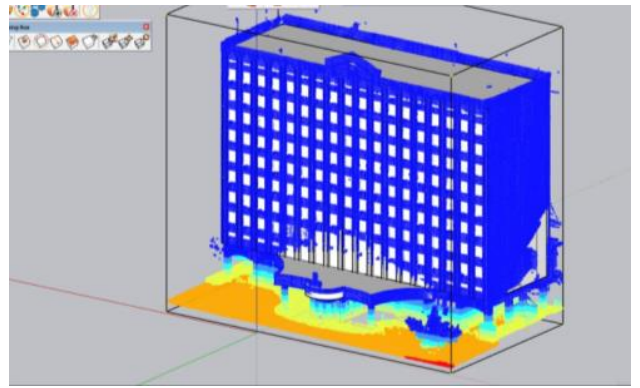

Figure 11. Display functions.

Undet for SketchUp has powerful point cloud data binding (fig.12). It is an irreplaceable push/pull function for maximum simulation fidelity. 


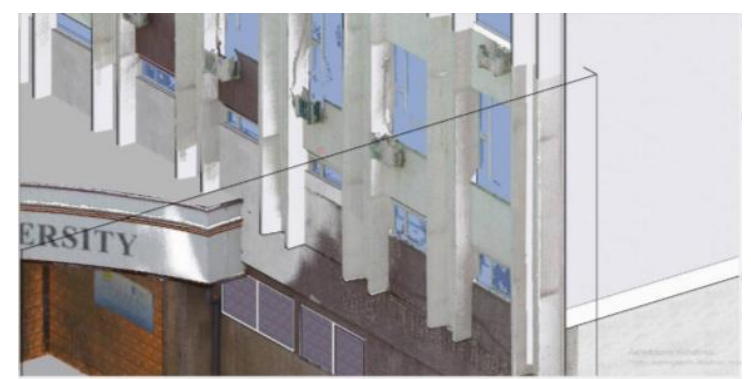

Figure 12. Binding function.

Turning on the snapping, with the help of a pencil, the parts of the building we needed were drawn over the point cloud. Because Satbayev University has a non-standard shape - I had to draw separately each part that differs from each others. The result is a complete and reliable model of the building (fig.13).

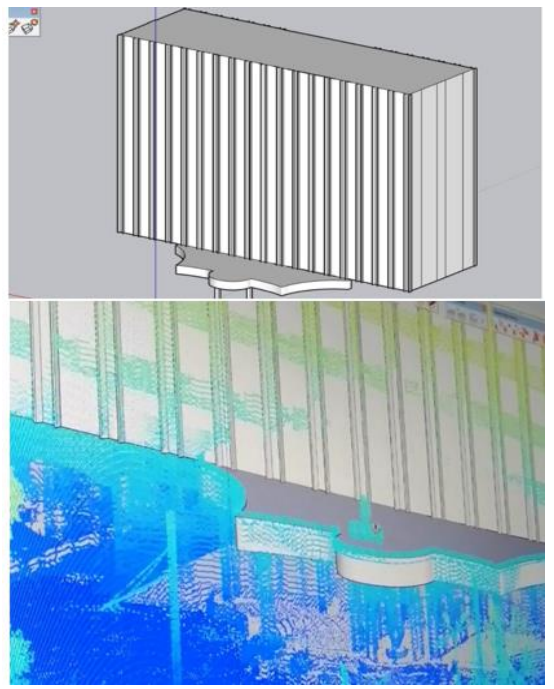

Figure 13. Modeling process.

After all parts of the building were ready, they were soldered together. An integral part of the work was drawing windows and doors in the model (fig.14). To do this, first, along the contour of the point cloud data, we outlined the windows, and using the "extrusion" function, they gave the shape of the places we need.

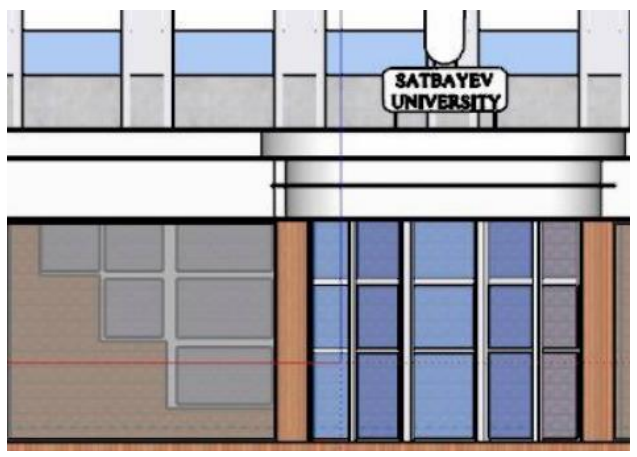

Figure 14. The process of extruding windows.

\subsection{Visualization problem}

To fully render the model, a texture was set. The realism of a 3D model directly depends on the choice of material from which the texture will be created. But in the process, the problem of reliable rendering arose due to a shortage of materials in the library, caused by a limited number of preset textures and colors of the standard palette. Therefore, it was decided to import the textures we need by the settings in the "Material" tab (fig. 15). However, GIF textures are not supported. Files are uploaded in the following formats: skp, png, jpg.

To give a surface texture, it is necessary to catch on one of the edges, the second on the opposite. Alternatively, you can drag and drop an image from the explorer, but for this, the "Use as image" option must be enabled. The finished model of the Satbayev university case after solving problems with the texture is shown in Figure 15.

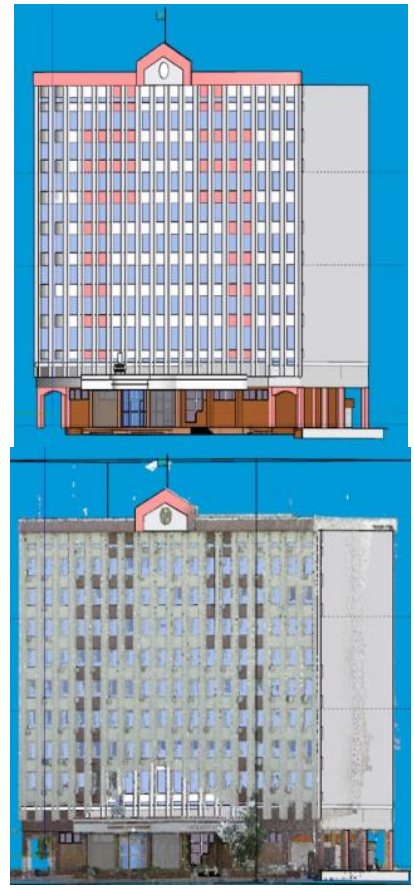

Figure 15. The result of 3D modeling of the building of Satbayev University.

\section{CONCLUSION}

This paper presents the detailed algorithms used by us for studying an object using UAVs and ground-based laser scanning. A comparative analysis based on the results of this study showed the following advantages and disadvantages of each of the methods.

In the case of using a UAV (DJI Phantom Development Team, 2017), the model was built according to the data obtained during the shooting of a dense cloud of points (Leica Cyclone REGISTER 360, 2017). The method is characterized by the following indicators:

$\checkmark \quad$ a sufficient level of detail for large objects, but objects;

$\checkmark \quad$ centimeter accuracy; deadlines);

$\checkmark \quad$ ensure the safety of surveyors when working at hazardous sites. 
In the case of laser scanning, the model is built using an algorithm for processing stereo aerial photographs obtained with digital cameras. This method has the following indicators compared to UAVs:

$$
\begin{array}{ll}
\checkmark & \text { high level of detail (higher point density); } \\
\checkmark & \text { the possibility of obtaining millimeter accuracy; } \\
\checkmark & \text { takes more time to shoot; } \\
\checkmark & \text { more complex organization of processing, including }
\end{array}
$$$$
\text { a limited number of software for post-processing. }
$$

However, compared with other types of laser scanners, it should be noted the advantages of the RTC 360 laser scanner used for this work, namely, high point density, fast shooting, more automated organization of shooting.

In general, it can be concluded that for the rapid construction of a model using simple processing algorithms and without setting high requirements for accuracy, the best solution is to shoot a UAV using the RTK mode. This solution is relevant for shooting large objects and provides a sufficient level of detail and accuracy (Rylsky I.A., 2017).

Terrestrial laser scanning should be used when the task is to achieve a high level of detail and accuracy of an object.

Shortly, the capabilities and accuracy of each of these methods will expand, leading to its successful application in research and production purposes.

\section{REFERENCES}

A. A. Baltiyeva, A. S. Raskaliyaev, A. I. Samsonenko, L. S. Shamganova, H. Fan., 2020: Development of the software and technical complex of the high-precision satellite positioning system in the conditions of open-pit mining processes. Kompleksnoe Ispol'zovanie Mineral'nogo Syr'a. = Complex Use of Mineral Resources = Mineraldik Shikisattardy Keshendi Paidalanu. № 4 (315), 42-48. (In Eng.). doi.org/10.31643/2020/6445.35

Copyright Leica Geosystems AG, 2017. 9435 Heerbrugg, Switzerland. All rights reserved. Printed in Switzerland. Leica Geosystems AG is part of Hexagon AB. 864328en - 11.17

DJI Phantom Development Team, 2017: Application of DJI Phantom 4 Pro RTK/PPK for design and construction in Switzerland. Open Source Geospatial Foundation. topodrone.ru/news/article/primenenie-dji-phantom-4-pro-rtkppk-dlya-proektirovaniya-i-stroitelstva-v-shveytsarii (23 December 2021)

Kuzmenko S.V., Shamganova L.S., Akhmedov D.Sh., Baltieva A.A., 2018: Information and navigation support for mining operations in the open pits of the Sokolovsko-Sarbai Mining and Processing Production Association. Mining Journal, No. 5, JSC Publishing House Ore and Metals. ISSN 00172278. DOI 10.17580/gzh.2018.05.11

Leica Cyclone REGISTER 360, 2017: Software for registering point clouds in 3D laser scanning. Open Source Geospatial Foundation. https://leica-geosystems.com/ru/products/laserscanners/software/leica-cyclone/leica-cyclone-register-360 (22 December 2021)

Leica ScanStation P30, 2015: Basic Training Manual. Basic Workflow Chart, 3-4.
Rylsky I.A., 2017: Comparison of the suitability of airborne laser scanning data and aerial photography from UAVs for design work. Open Source Geospatial Foundation. russiandrone.ru/publications/sravnenie-prigodnosti-dannykhvozdushnogo-lazernogo-skanirovaniya-i-aerofotosmki-sbpla-dlya-obespech (12 October 2021)

Service Geo, 2014. Creation of 3D models using aerial photography Open Source Geospatial Foundation. srvgeo.ru/services/aerofotosemka/sozdanie-3d-modelejmetodom-aerofotosemki (20 December 2021)

Undet for SketchUp Development Team, 2020. A feature-rich point cloud extension to create 3D models based on scan data. Open Source Geospatial Foundation. undet.com/undetproducts/sketchup-plugin-for-3d-modeling-from-point-cloud (20 December 2021)

Yuneec, 2019. Top 10 programs for creating 3D models and maps using drones. Open Source Geospatial Foundation. yuneecrussia.ru/10-best-software-3dmapping (22 December 2021) 